\title{
Social and cognitive functioning, urbanicity and risk for schizophrenia
}

\author{
MARK WEISER, JIM VAN OS, ABR AHAM REICHENBERG, \\ JONATHAN RABINOWITZ, DANIELLA NAHON, EFRAT KRAVITZ, \\ GAD LUBIN, MOTI SHMUSHKEVITZ, HAIM Y. KNOBLER, SHLOMO NOY \\ and MICHAEL DAVIDSON
}

\begin{abstract}
Background Previous work suggests that the association between urbanicity and schizophrenia may be greatest in those with pre-existing vulnerability.
\end{abstract}

\begin{abstract}
Aims To test for synergism in risk of schizophrenia between population density and a combined exposure of poor premorbid social and cognitive functioning.
\end{abstract}

Method For 371 603 adolescent males examined by the Israeli Draft Board on social and cognitive functioning, data on population density of place of residence and later hospitalisation for schizophrenia were obtained from population-based registries.

\section{Results There was an interaction between population density (five levels) and poor premorbid social and cognitive functioning (interaction $\chi^{2}=4.6, P=0.032$ ). The adjusted increase in cumulative incidence associated with one unit change in population density was $0.10 \%$ in the vulnerable group $(95 \% \mathrm{Cl} 0.019-0.18$, $P=0.015)$, nine times larger than that in the non-vulnerable group $(0.011 \%, 95 \% \mathrm{Cl}$ $0.0017-0.020, P=0.021)$.}

Conclusions Risk of schizophrenia may increase when people with a genetic liability to the disorder, expressed as poor social and cognitive functioning, need to cope with city life.

Declaration of interest None.
The factors responsible for the wellestablished association between urban dwelling and increased rate of schizophrenia remain unclear (Lewis et al, 1992; Haukka et al, 2001; Pedersen \& Mortensen, 2001a; Peen \& Dekker, 2004; van Os, 2004), but may represent an interaction between genetic vulnerability and the urban environment (Lewis et al, 1992; Haukka et al, 2001; Pedersen \& Mortensen, 2001a; Peen \& Dekker, 2004; van Os, 2004). The aim of our study was to investigate possible interactions between poor premorbid cognitive and social functioning, and urban living, on risk of schizophrenia. In order to do this we cross-linked information from three databases: data from the nationwide screening of the entire population of male adolescents at age 16-17 years performed by the Israeli Draft Board; data from the Israeli Central Bureau of Statistics, which uses census data to ascertain population density according to residence; and data on hospitalisations for schizophrenia from the Israeli National Psychiatric Hospitalisation Registry.

\section{METHOD}

The sample consisted of a population-based cohort of 376623 Israeli-born male adolescents, assessed by the Israeli Draft Board at age 16-17 years. As immigrants are more likely to live in urban than in rural areas (Dey \& Lucas, 2006), and immigrants have been reported to be at increased risk of schizophrenia (Cantor-Graae \& Selten, 2005), we accounted for possible effects of immigration by examining only nativeborn Israelis.

\section{Assessment}

At age 16-17 years all Israeli males undergo cognitive, behavioural and psychiatric assessments by the draft board in order to determine their eligibility and aptitude for military service. The cognitive assessment includes Raven's Progressive Matrices Revised, which consist of a series of visual pattern matching and analogy problems. This test measures non-verbal abstract reasoning and visual-spatial problem-solving abilities, is highly correlated with general cognitive abilities (Duncan et al, 2000), is scored between 0 (lowest) to 30 (highest) and has a normal distribution. After the cognitive assessments are performed, an interview assessing personality and behavioural traits is administered by trained college-age individuals who have completed a 4-month training course on administration of the interview. The behavioural assessment includes a sub-scale assessing social functioning; this assessment includes questions such as 'How many good friends do you have?', 'Do you have a girlfriend?' and 'Do you generally prefer to be with or without a group of companions?'. Scale points are 1, very poor: complete withdrawal; 2, poor: weak interpersonal contacts; 3, adequate: can form relationships with individuals and in a group; 4, good: good interpersonal relationships; and 5, exceptional: superior interpersonal relatedness. The test-retest reliability of the behavioural assessment for inductees interviewed after several days by different interviewers is above 0.8 , and population-based norms are available (Gal, 1986).

On the basis of the interview and a physician's examination, adolescents who might be suffering from behavioural disturbances or mental illness are referred for an in-depth assessment by a mental health professional, and if the adolescent warrants a psychiatric diagnosis, a board-certified psychiatrist will examine him. Criteria for referral for an in-depth mental health assessment are having the lowest score on the prediction sub-scale (which reflects the interviewer's assessment of the adolescent's ability to succeed in the military), a history of psychological or psychiatric treatment, current complaints, or manifestation of behavioural abnormalities during the assessment procedure. The mental health assessment is done using a semi-structured interview administered by a clinical social worker or psychologist, who enquires about personal and family history, previous psychological and psychiatric treatments, interpersonal relationships, self-esteem, self-injurious and antisocial acts, and functioning within the family and in school. If the clinician suspects that the adolescent has psychopathological symptoms, the adolescent is referred to a board-certified 
psychiatrist for evaluation and an ICD-9 diagnosis. For a more detailed description of the draft board assessment procedure, see Gal (1986) and Tubiana \& Ben-Shachar (1982).

\section{Israeli Central Bureau of Statistics}

The Israeli Central Bureau of Statistics divides the country into 'geographical units', which are areas with 3000-4000 residents. The division is performed so that the population in each area is as homogeneous as possible in terms of ethnic background, culture and income. Information about population density (calculated as number of persons per $\mathrm{km}^{2}$ of each geographical unit) was obtained, as was a measure of socio-economic status, based on number of persons per room in the home, number of computers per household, number of motor vehicles per household and per capita income (Central Bureau of Statistics, 1995)

\section{Israeli Psychiatric Hospitalisation Case Registry}

The Israeli Psychiatric Hospitalisation Case Registry is a complete listing of all ICD-10 discharge diagnoses assigned by a boardcertified psychiatrist at the reporting facility. All psychiatric hospitals, day hospitals and psychiatric units in general hospitals are required by law to report all admissions and discharges to this registry. From the registry we identified patients with a last discharge diagnosis of schizophrenia (ICD-10 codes F20.0-F20.9).

\section{Study population}

The file containing data on population density by address of residence at the time of draft board assessment was linked to the draft board file, which contains results of the board's assessments for the entire national population of adolescents. This file was in turn linked to the Israeli National Psychiatric Hospitalisation Case Registry, using national identification numbers (equivalent to the US social security number). Before the merged file was returned to the investigators for analysis, the national identification numbers were removed, leaving the merged file un-identified, in order to preserve confidentiality. This procedure identified 376623 Israeli-born male adolescents consecutively assessed by the draft board, with a (mean 8.57, s.d.=4.06) follow-up period for psychiatric hospitalisation of 1-17 years. From this file, we excluded $2251(0.6 \%)$ inductees who had been diagnosed during the draft board assessment as having a psychotic disorder or major affective disorder (as some of these adolescents had major affective disorder with psychotic symptoms). In addition, in order to exclude individuals who had existing psychotic illness, or who had possibly been in the prodromal phase of their illness when assessed by the draft board, we excluded 717 (0.2\%) persons who had been hospitalised before or up to 1 year after the draft board assessment. Because this analysis referred only to people later hospitalised with schizophrenia, we also excluded $2300(0.6 \%)$ adolescents who were later hospitalised with discharge diagnoses other than schizophrenia. Owing to overlap between the excluded groups, the file analysed contained data on 371603 male adolescents who were born in Israel, were found not to have a psychotic disorder as assessed by the draft board procedure, and had not been admitted to hospital for any psychiatric disorder before or within 1 year after the draft board assessment.

\section{Statistical analyses \\ Main effects}

Population density was categorised (van Os et al, 2003, 2004) into five levels by dividing the population into equal quintiles. Social functioning was divided into three groups: very poor or poor; adequate; and good or excellent. Cognitive functioning (reflected by the scores on the Raven's Progressive Matrices - Revised test) was categorised into three groups by dividing the population into equal thirds, reflecting high, intermediate and low functioning. As previous studies have shown poor social functioning and poor cognitive functioning to be independently associated with increased risk of later schizophrenia (Davidson et al, 1999), we defined adolescents with both poor social and poor cognitive functioning as having high vulnerability, compared with the rest of the population.

We first estimated the individual associations between population density and high vulnerability using Cox regression models, taking into account the amount of time of follow-up until hospitalisation, or the date that the military file was linked to the hospitalisation file. We examined the effect of being vulnerable and of population density on the risk of hospitalisation for schizophrenia, while controlling for the other factor and the potential confounding effect of socio-economic status.

\section{Interaction effects}

Biological synergism (co-participation of causes towards the same outcome) between environmental risk and background vulnerability is thought to be common in multifactorial disorders such as schizophrenia. The classic problem, however, is how biological synergism can be inferred from statistical manipulations with research data (statistical interaction), in particular with regard to the choice of additive or multiplicative models. It has been shown that the true degree of biological synergism can be better estimated from - but is not the same as - the additive statistical interaction (Darroch, 1997; Murray, 2003). This new method was recently applied to schizophrenia, showing synergy between traumatic head injury and familial liability (Malaspina et $a l, 2001$ ) between cannabis and psychosis liability (van Os et al, 2002) and between urbanicity and proxy genetic risk factors (van Os et al, 2003, 2004; Spauwen et al, 2006). Conforming to these previous publications, we calculated the additive interaction between social and cognitive vulnerability on the one hand, and population density on the other, in models of schizophrenia. The statistical method used was similar to that used in other recent publications on this topic (van Os et al, 2003, 2004; Spauwen et al, 2006) in that effects were expressed on the additive scale (i.e. as a risk difference rather than a risk ratio), using risk difference regression in Stata, version 9.1. The risk difference regression procedure in Stata fits generalised linear models estimating risk differences (Wacholder, 1986; Hardin \& Cleves, 1999). The statistical significance of the interactions was assessed by Wald test (Clayton \& Hills, 1993). After calculation of the interaction term, effect sizes of population density, stratified by level of vulnerability, were calculated from the model using the appropriate linear combinations with the Stata LINCOM command. All analyses were controlled for age and socioeconomic status.

\section{RESULTS}

Of the 371603 non-psychotic male adolescents assessed by the Draft Board at age 16-17 years and followed for hospitalization for schizophrenia over 1-17 years, $1174(0.3 \%)$ were later hospitalised for 
schizophrenia. While controlling for age and socio-economic status, increasing population density (adjusted hazard ratio (HR) over five levels 1.07, 95\% CI 1.031.11) and vulnerability $(\mathrm{OR}=3.34,95 \%$ CI 2.90-3.84) were associated with increased risk of later schizophrenia, independently of each other (Table 1). The Pearson correlation between population density and vulnerability to schizophrenia was very small (0.0098), indicating that the variables making up the interactions were independent of each other, so that the hypothesis of moderation can be distinguished from effects occasioned by mediation. The Pearson correlation between social and cognitive functioning was somewhat larger, at 0.15 .

The effect of increasing population density was larger for vulnerable compared with non-vulnerable individuals (interaction $\chi^{2}=4.6, P=0.032$ ). Stratified analyses revealed that the risk difference per unit change in population density was $0.011 \%$ in the non-vulnerable group $(95 \% \mathrm{CI}$ $0.0016-0.020, P=0.021$ ), whereas in the vulnerable group it was nearly ten times larger (risk difference per unit change in population density $0.10 \%, 95 \%$ CI $0.019-0.18, P=0.015)$, indicating that the effect of increasing population density on increasing risk of schizophrenia is particularly relevant for adolescents with both

Table I Population density, social functioning and cognitive functioning, and cumulative incidence of schizophrenia

\begin{tabular}{lrc}
\hline Variable & $n$ & $\begin{array}{c}\text { Cases of } \\
\text { schizophrenia } \\
n(\%)\end{array}$ \\
& & \\
\hline $\begin{array}{l}\text { Population density } \\
\text { Low }\end{array}$ & 73984 & $181(0.2)$ \\
Low-intermediate & 74282 & $216(0.3)$ \\
Intermediate & 74435 & $247(0.3)$ \\
Intermediate-high & 74263 & $258(0.3)$ \\
High & 74639 & $272(0.4)$ \\
Social functioning & & \\
Low & 65079 & $457(0.70)$ \\
Intermediate & 208545 & $559(0.27)$ \\
High & 75178 & $105(0.14)$ \\
Cognitive functioning & & \\
Low & 122395 & $650(0.53)$ \\
Intermediate & 118644 & $314(0.26)$ \\
High & 130560 & $210(0.16)$ \\
\hline
\end{tabular}

poor social and poor cognitive functioning (Table 2).

\section{DISCUSSION}

The main finding of this historical prospective analysis is that the effect of living in areas of increasing population density increases the risk of later hospitalisation for schizophrenia in men with vulnerability for schizophrenia, expressed as poor social and cognitive abilities. As social and cognitive abilities are at least partially affected by genetic factors (Kendler et al, 1991; Plomin, 1999), these results might be interpreted to represent a gene-environment interaction affecting risk of schizophrenia, and might provide an insight into the mechanism of the well-replicated association between urban dwelling and risk of schizophrenia (Krabbendam \& van Os, 2005). Past research indicates that impairments in social functioning (Kendler et al, 1982) and cognitive impairment (Szoke et al, 2005) are associated with genetic risk of schizophrenia. Living in a city is likely to be a proxy for an environmental influence such as stress, use of illegal drugs, poverty, crowding or other effects yet unknown. The gene-environmental interaction might occur when people at genetic risk of schizophrenia, expressed as poor social and cognitive abilities, experience stress when confronted with the need for relatively intense and more ambiguous social interactions and decision-making (based on cognitive factors) typical in large cities. This is supported by the finding of an association between stress and later schizophrenia (Mazure, 1995). These data are also supported by studies that have demonstrated that people with a family history of psychotic disorder - who often have poor social (Laurent et al, 2000) and cognitive functioning (Cannon et al, 2000) - who live in cities, are at particularly high risk of later schizophrenia (van Os et al, 2003, 2004). Investigators (e.g. Freeman, 1994) have suggested that the association between urbanicity and schizophrenia is due to increased numbers of immigrants in cities. As we limited our analysis to native-born Israelis and still found an increased risk of schizophrenia in areas of higher population density, it seems that immigrants in cities are not the cause of this association.

As the initial analysis of the effect of population density was controlled for social and cognitive functioning, the results presented here indicate there is still an independent effect of increasing population density on risk of schizophrenia. This finding is unique, as, to the best of our knowledge, none of the previous studies on the effect of population density of risk

Table 2 Comparison of the effect of population density in people with low and high vulnerability for schizophrenia

\begin{tabular}{lccc}
\hline Population density & $n$ & $\begin{array}{c}\text { Schizophrenia } \\
\text { cumulative } \\
\text { prevalence } \\
\%(n)\end{array}$ & $\begin{array}{c}\text { Adjusted risk difference per unit increase' } \\
\%(95 \% \mathrm{Cl})\end{array}$ \\
\hline Non-vulnerable & & & $0.11(0.0017-0.020)$ \\
Low density & 59396 & $0.22(133)$ \\
Low-intermediate & 66249 & $0.23(152)$ \\
Intermediate & 66169 & $0.27(180)$ & \\
Intermediate-high & 64745 & $0.30(192)$ & \\
High density & 61964 & $0.31(191)$ & \\
Vulnerable & & $0.10(0.019-0.018)$ \\
Low density & 4963 & $0.60(30)$ & \\
Low-intermediate & 6108 & $0.94(58)$ & \\
Intermediate & 6254 & $0.96(61)$ & \\
Intermediate-high & 6207 & $0.96(60)$ & $1.11(64)$ \\
High density & 5625 & &
\end{tabular}

Interaction: $\chi^{2}=4.6$, d.f. $=\mathrm{I}, P=0.032^{2}$

I. All analyses controlled for age and socio-economic status.

2. Interaction tests whether the risk difference per unit increase in the vulnerable group is significantly greater than the risk difference per unit increase in the non-vulnerable group. 
for schizophrenia controlled for social functioning.

As the analyses controlled for socioeconomic status, this report also confirms previous findings that socio-economic position prior to disease onset is not the cause of the association between schizophrenia and urban dwelling (Harrison et al, 2003).

\section{Limitations}

A previous report on the same topic reported absence of interaction between urbanicity and family history (Mortensen et al, 1999). That report, however, was based on calculation of the multiplicative interaction, and it is important to note that absence of interaction between two covariates on the multiplicative level, with the exception of some special cases, will result in presence of interaction between these covariates on the additive level, and vice versa. This distinction is important, as it has recently been shown that the degree of biological synergism can be more readily deduced from the additive interaction (Darroch, 1997). Thus, had we calculated interaction under the multiplicative model using Cox regression, we would not have found significant interaction $\left(\chi^{2}=0.8\right.$, $P=0.37$ ), although also under the multiplicative model the risk per unit increase in urbanicity would have been more than $80 \%$ greater in the vulnerable group $(\mathrm{HR}=1.11,95 \%$ CI $1.02-1.21, P=0.020)$ compared with the non-vulnerable group $(\mathrm{HR}=1.06,95 \%$ CI $1.01-1.11, P=0.020)$, adding to the validity of our findings.

Since young women do not undergo the systematic behavioural and psychiatric assessment by the draft board, our findings apply directly only to men. Other research, however, has shown that urbanicity increases the risk of psychosis in both men and women (Peen \& Dekker, 2003; Sundquist et al, 2004; Krabbendam \& van Os, 2005). Also, using population density as such does not allow one to differentiate completely between urban and rural areas, as there are neighbourhoods with relatively low population density in urban areas, and neighbourhoods with relatively high population density in rural areas. This, however, has advantages as well, since being an actual measure of the number of people per unit area, it permits the assessment of the influence of population density as such, without making assumptions regarding the definitions of 'rural' and 'urban'.
Another limitation is that our results were not adjusted for parental mental illness (data that were not available to us). However, when other authors adjusted for this potential confounder, the urbanisation effect remained (Pedersen \& Mortensen, $2001 b$ ). Also, neither the military database nor the psychiatric hospitalisation registry includes data regarding loss to follow-up, i.e. death or emigration. However, as the period of follow-up was 8.6 years (s.d.=4.1), until approximate age 25-26 years, death should not be a major cause of loss to follow-up, as the rates of death in this age group are slightly less than 1/ 1000 per year (information from the Israeli Central Bureau of Statistics). We were not able to find data on emigration from Israel during the years relevant to this study.

Another potential limitation is that the case registry diagnoses are clinical rather than research diagnoses. However, these diagnoses were assigned by board-certified psychiatrists who had the benefit of observing the patient throughout one or more hospitalisations, and had been trained and re-trained in the use of the diagnostic criteria of the ICD-9 and ICD-10. Moreover, studies that have compared clinical diagnoses of schizophrenia assigned in state hospitals (Pulver et al, 1988) with research diagnoses have shown a high degree of concordance between them. In a study published by our group we found that, compared with research diagnoses established using a Schedule for Affective Disorders and Schizophrenia - Lifetime interview, the registry diagnoses of schizophrenia had a sensitivity of 0.89 (Weiser et al, 2005). Even if diagnostic misclassification had been an issue, this would have served to increase random error, making it more difficult to find an association between population density and schizophrenia, rather than producing a spurious one.

\section{Strengths}

To the best of our knowledge, this is the first study to use a direct measure of social functioning to assess its interaction with the association between increasing population density and risk of schizophrenia, and is similar to a previous study measuring the quantity of social interaction, which reported similar results (Stefanis et al, 2004). Also, while assessing the effect of increasing population density on risk of later hospitalisation for schizophrenia, we removed the data for adolescents diagnosed with a psychotic disorder in the draft board assessment, and those hospitalised for psychotic disorder before or within a year after the assessment. This ensured that these data were not confounded by people with a (prodromal) psychotic disorder moving from rural to urban areas. We also used multiple data-sets compiled independently of the research hypotheses, and had a relatively long follow-up period of a populationbased sample.

\section{REFERENCES}

Cannon, T. D., Huttunen, M. O., Lonnqvist, J., et al (2000) The inheritance of neuropsychological dysfunction in twins discordant for schizophrenia. American Journal of Human Genetics, 67, 369-382.

Cantor-Graae, E. \& Selten, J. P. (2005) Schizophrenia and migration: a meta-analysis and review. American Journal of Psychiatry, 162, 12-24.

Central Bureau of Statistics (1995) Demographic Characteristics of the Population in Localities and Statistical Areas. Israel: Ministry of the Interior.

Clayton, D. \& Hills, M. (1993) Wald tests. In Statistical Models in Epidemiology (eds D. Clayton \& M. Hills), pp. 101-102. Oxford Science Publications.

Darroch, J. (1997) Biologic synergism and parallelism. American Journal of Epidemiology, 145, 661-668.

Davidson, M., Reichenberg, A., Rabinowitz, J., et al (1999) Behavioral and intellectual markers for schizophrenia in apparently healthy male adolescents. American Journal Psychiatry, 156, 1328-1335.

Dey, A. N. \& Lucas, J.W. (2006) Physical and mental health characteristics of US- and foreign-born adults: United States, 1998-2003. Advance Data, 369, I-19.

Duncan, J., Seitz, R. J., Kolodny, J., et al (2000) A neural basis for general intelligence. Science, $\mathbf{2 8 9}$ 457-460.

Freeman, H. (1994) Schizophrenia and city residence British Journal of Psychiatry, 164 (suppl. 23), 39-50.

Gal, R. (1986) The selection, classification and placement process. In A Portrait of the Israeli Soldier, p. 77. Greenwood Press.

Hardin, J. \& Cleves, M. (1999) Generalized linear models: extensions to the binomial family: seb29. Stata Technical Bulletin, 50, 2I-25.

Harrison, G., Fouskakis, D., Rasmussen, F., et al (2003) Association between psychotic disorder and urban place of birth is not mediated by obstetric complications or childhood socio-economic position: a cohort study. Psychological Medicine, 33, 723-731.

Haukka, J., Suvisaari, J., Varilo, T., et al (200I)

Regional variation in the incidence of schizophrenia in Finland: a study of birth cohorts born from 1950 to 1969. Psychological Medicine, 3I, 1045-1053.

Kendler, K. S., Gruenberg, A. M. \& Strauss, J. S. (1982) An independent analysis of the Copenhagen sample of the Danish Adoption Study of Schizophrenia. $\mathrm{V}$. The relationship between childhood social withdrawal and adult schizophrenia. Archives of General Psychiatry, 39, $1257-126 \mid$.

Kendler, K. S., Ochs, A. L., Gorman, A. M., et al (1991) The structure of schizotypy: a pilot multitrait twin study. Psychiatry Research, 36, 19-36. 
Krabbendam, L. \& van Os, J. (2005) Schizophrenia and urbanicity: a major environmental influence conditional on genetic risk. Schizophrenia Bulletin, $\mathbf{3 1}$ 795-799.

Laurent, A., Biloa-Tang, M., Bougerol, T., et al (2000) Executive/attentional performance and measures of schizotypy in patients with schizophrenia and in their nonpsychotic first-degree relatives. Schizophrenia Research, 46, 269-283.

Lewis, G., David, A., Andreasson, S., et al (1992) Schizophrenia and city life. Lancet, 340, 137-140.

Malaspina, D., Goetz, R. R., Friedman, J. H., et al (200I) Traumatic brain injury and schizophrenia in members of schizophrenia and bipolar disorder pedigrees. American Journal of Psychiatry, 158, 440-446.

Mazure, C. M. (1995) Does Stress Cause Psychiatric Illness? American Psychiatric Press.

Mortensen, P. B., Pedersen, C. B., Westergaard, T., et al (1999) Effects of family history and place and season of birth on the risk of schizophrenia. New England Journal of Medicine, 340, 603-608.

Murray, R. (2003) The Epidemiology of Schizophrenia. Cambridge University Press.

Pedersen, C. B. \& Mortensen, P. B. (200la) Evidence of a dose-response relationship between urbanicity during upbringing and schizophrenia risk. Archives of General Psychiatry, 58, 1039-1046.

Pedersen, C. B. \& Mortensen, P. B. (200Ib) Family history, place and season of birth as risk factors for schizophrenia in Denmark: a replication and reanalysis. British Journal of Psychiatry, 179, 46-52.

Peen, J. \& Dekker, J. (2003) Urbanisation as a risk indicator for psychiatric admission. Social Psychiatry and Psychiatric Epidemiology, 38, 535-538.

Peen, J. \& Dekker, J. (2004) Is urbanicity an environmental risk-factor for psychiatric disorders? Lancet, 363, 2012-2013.

Plomin, R. (1999) Genetics and general cognitive ability. Nature, 402, C25-C29.

Pulver, A. E., Carpenter, W. T., Adler, L., et al (1988) Accuracy of the diagnoses of affective disorders and

MARK WEISER, MD, Department of Psychiatry, Sheba Medical Center, Tel-Hashomer, IDF, Division of Menta Health, and Sackler School of Medicine, Tel Aviv University, Ramat Aviv, Israel; JIM VAN OS, Department of Psychiatry and Neuropsychology, Maastricht University, The Netherlands, and Division of Psychological Medicine, Institute of Psychiatry, London, UK; ABRAHAM REICHENBERG, PhD, Department of Psychiatry, Mount-Sinai School of Medicine, New York, USA; JONATHAN RABINOWITZ, PhD, Bar llan University, Ramat Gan, Israel; DANIELLA NAHON, MA, Department of Mental Health, Ministry of Health, Jerusalem, Israel; EFRAT KRAVITZ, BSc, Department of Psychiatry, Sheba Medical Center, Tel-Hashomer, Israel; GAD LUBIN, MD, MOTI SHMUSHKEVITZ, MD, HAIM Y. KNOBLER, MD, Division of Mental Health, Sheba Medical Center, Tel-Hashomer, Israel; SHLOMO NOY, MD, PhD, MICHAEL DAVIDSON, MD, Department of Psychiatry, Sheba Medical Center, Tel-Hashomer and Sackler School of Medicine, Tel Aviv, University, Ramat Aviv, Israel

Correspondence: Mark Weiser, MD, Department of Psychiatry, Sheba Medical Center, Tel-Hashomer, Israel 5262I.Email: mweiser@netvision.net.il

(First received 3 October 2006, final revision 3 May 2007, accepted 14 May 2007)

schizophrenia in public hospitals. American Journal of Psychiatry, 145, 218-220.

Spauwen, J., Krabbendam, L., Lieb, R., et al (2006) Evidence that the outcome of developmental expression of psychosis is worse for adolescents growing up in an urban environment. Psychological Medicine, 36, 407-4I5.

Stefanis, N. C., Delespaul, P., Smyrnis, N., et al (2004) Is the excess risk of psychosis-like experiences in urban areas attributable to altered cognitive development? Social Psychiatry and Psychiatric Epidemiology, 39, 364-368.

Sundquist, K., Frank, G. \& Sundquist, J. (2004)

Urbanisation and incidence of psychosis and depression: follow-up study of 4.4 million women and men in Sweden. Bitish Journal of Psychiatry, 184, 293-298.

Szoke, A., Schurhoff, F., Mathieu, F., et al (2005) Tests of executive functions in first-degree relatives of schizophrenic patients: a meta-analysis. Psychological Medicine, 35, 77I-782.

Tubiana, J. H. \& Ben-Shachar, G. (1982) An objective group questionnaire as a substitute for a personal interview in the prediction of success in military training in Israel. Personal Psychology, 35, 349-357. van Os, J. (2004) Does the urban environment cause psychosis? British Journal of Psychiatry, 184, 287-288.

van Os J., Bak, M., Hanssen, M., et al (2002)

Cannabis use and psychosis: a longitudinal populationbased study. American Journal of Epidemiology, I56, 319-327.

van Os, J., Hanssen, M., Bak, M., et al (2003) Do urbanicity and familial liability coparticipate in causing psychosis? American Journal of Psychiatry, 160, 477-482.

van Os, J., Pedersen, C. B. \& Mortensen, P. B. (2004) Confirmation of synergy between urbanicity and familial liability in the causation of psychosis. American Journal of Psychiatry, 161, 2312-2314.

Wacholder, S. (1986) Binomial regression in GLIM: estimating risk ratios and risk differences. American Journal of Epidemiology, 123, 174-184.

Weiser, M., Kanyas, K., Malaspina, D., et al (2005) Sensitivity of ICD-10 diagnosis of psychotic disorders in the Israeli National Hospitalization Registry compared with RDC diagnoses based on SADS-L. Comprehensive Psychiatry, 46, 38-42. 